\title{
Key Questions and Technology in Clinical Practice
}

This month we bring our readers some key questions related to mental health, ${ }^{1-4}$ health literacy, ${ }^{5}$ osteoporosis, $^{6}$ and mandatory vaccination. ${ }^{7} \mathrm{We}$ also have reports of some good use of technology for asthma care ${ }^{8,9}$ and for women's care,${ }^{10,11}$ as well as some good images in our case report ${ }^{11}$ and clinical review. ${ }^{12}$ Winters et $\mathrm{al}^{13}$ and Crosson et $\mathrm{al}^{14}$ discuss issues related to improving cardiovascular risk factors, including the importance of the usual source of care and the frustrations of the physicians who are the usual source of care.

\section{Key Technology for Asthma: Automated Telephone Intervention and Peak Flow Meters}

Using an automated telephone intervention, including at least 2 phone calls during 10 weeks, Bender et $\mathrm{al}^{8}$ increased patient adherence to asthma controller medications, with approximately two thirds of the patients in the intervention group adherent at the end of the trial. Although there was no long-term follow-up, this study encourages us to use this technology for patients with asthma. Meanwhile, McCoy et $\mathrm{al}^{9}$ have made our lives simpler: a sitting peak flow measurement is as good as a standing peak flow measurement.

\section{Key Question: Should We Mandate the Human Papillomavirus Vaccine?}

Would requiring Gardasil (Merck \& Co., Whitehouse Station, NJ), the human papillomavirus (HPV) vaccine, for school attendance increase the uptake and completion rate, which is currently low? School requirements have increased vaccination rates of other vaccines. Ferris et $\mathrm{al}^{7}$ explore the thoughts of parents about requiring the HPV vaccine for school attendance. Most parents indicate that it should not be mandatory. However, those who believed the vaccine prevented warts or cervical cancer and wanted it for their daughter were much more likely to express support for the mandatory program. This suggests that increasing parental knowledge about the benefits of this vaccine

Conflict of interest: The authors are editors of the FABFM. might increase support for a mandatory program. Furthermore, if the vaccine were required most parents would agree to have their child vaccinated.

\section{Key Instrument: The Fischer Cone Biopsy Excisor}

For patients in whom the HPV vaccine fails or who never received it, any cervical dysplasia needs treatment. Mulhem et al ${ }^{10}$ report good results using the Fischer cone biopsy excisor for cervical dysplasia, including those procedures done by family medicine residents under an attending physician's supervision. This Fischer cone biopsy excisor is distinct from the loop electrosurgical excision procedure in that it provides greater stabilization for the excisor wire.

\section{Key Questions about Mental Health}

We have 4 articles exploring mental health issues, and each provides key questions or concepts we should use when discussing mental health with patients.

\section{Fatigue?}

Fatigue and stress are very common, and most patients report that they have stress. So how do we determine when stress is sufficient enough to cause poor health? The answer to this is basically when stress is high enough to cause fatigue. Fatigue is a warning sign that indicates harmful levels of stress. Table 1 in the article by Maghout-Juralti et $\mathrm{al}^{2}$ is central to showing the multiple relationships. When stress was not related to fatigue there was no impact of the amount of reported stress on perceived health. Although sleeping problems were also related to fatigue, it was still the fatigue itselfnot insomnia - that was most important. Thus, our key question is about fatigue.

\section{Depression?}

Are you depressed? Do you believe in the personal ability to improve sleep? Bluestein et $\mathrm{al}^{4}$ further tease out how family physicians can work to help their patients with insomnia. As noted by Maghout-Juralti, ${ }^{2}$ insomnia was associated with worse 
health status. However, the areas for attack in the Bluestein et $\mathrm{al}^{4}$ study included depression and low self-efficacy; attention to these issues could potentially improve insomnia. Office counseling techniques such as motivational interviewing for both insomnia and depression (possibly presenting concurrently) should be studied for their possible efficacy.

\section{Self-Injury?}

Nonsuicidal self-injury is not uncommon (present in $1 \%$ to $4 \%$ of adults); it is much more common than suicidal self-injury and the behavior gives relief from unpleasant emotions. It most often involves cutting, but other actions include skin carving, burning, severe abrading/scratching of the skin, and/or punching or hitting. ${ }^{3}$ It is particularly common among patients who have borderline personality disorder. Many adolescents who injure themselves are depressed. Kerr et $\mathrm{al}^{3}$ report that careful probing and questioning can help identify those who self-injure, which can then lead to potential treatment and prevention of recurrences. Increasing severity of self-injury and/or multiple episodes may presage suicide attempts.

\section{Empty/Worthless/Hopeless Versus Happy?}

Heisel et $\mathrm{al}^{1}$ found that a positive answer to even 1 of 5 diagnostic questions had a high degree of sensitivity and specificity for suicidal ideation in the elderly even though the questions were not explicitly about self-harm or suicidal intent. Rather, the questions were about feelings of emptiness, worthlessness, or hopelessness, or feelings of their opposite: happiness. These questions are markers to further explore potential self-harm. I (MAB) have only had one elderly patient commit suicide; it was a man whom I had never seen, but his wife told me enough that I suspected suicidal intent and had him see a psychiatrist. I had his wife take away all gunrelated items from the home but he had one hidden. He saw the psychiatrist once and denied suicidal intent, but then he committed suicide the day before his second scheduled appointment. I have always wondered how things might have been different if I had had the opportunity to have a longterm relationship with him. If he had been assessed for hopelessness rather than been directly asked about suicidal intent, could we have avoided this bad outcome?

\section{Key Question: Osteoporosis is Found in Men?}

Men get osteoporosis, too. However, we are not as clear about which men to screen and when. Shepherd et $\mathrm{al}^{6}$ used the 3-factor (think: men who are thin, older, and have a history of chronic obstructive pulmonary disease are at greater risk) Male Osteoporosis Risk Estimation Score (MORES) to estimate how well it performed in the identification of men who should be screened to help prevent vertebral fractures. MORES has been shown to reduce the number needed to screen to prevent hip fractures. Although MORES is better than universal screening, the results with MORES were that we would need to screen thousands of men to prevent one vertebral or one hip fracture. Is this sensible? Personally, I (MAB) think we need to have a stronger evidence base of benefits and harms before recommending screening based on this or other questionnaires.

Shepherd et $\mathrm{al}^{6}$ also note that osteoarthritis, which involves hypertrophy of the bone, can decrease the accuracy of bone densitometry, creating diagnostic problems in practice. We do need better tests and better data about screening. We know treatment works, now we just need to identify when and whom to screen.

\section{Key Question: Usual Source of Care?}

Winters et $\mathrm{al}^{13}$ report that having a usual source of care was strongly associated with eligible patients with high low-density lipoprotein cholesterol and who are taking statin drugs, but not with goal attainment. In addition to the potential for inadequate statistical power, I (MAB) suspect one of the reasons for this apparent discrepancy between taking statins and goal attainment is that low-density lipoprotein cholesterol is often well treated with just one pill a day compared with treatment for many other types of medical problems. The usual source of care helped patients get on treatment; thus, the major cut point is treatment versus no treatment. The generic statins available at the time of the data collection (such as lovastatin) and thus more likely to be used by those with less money were those that were modestly less effective. Further, when statins alone are not enough, the other types of issues for medication treatment-such as ability to pay for medications-become increasingly important. In addition, we should note that the study highlights that many eligible patients were not taking statins when they should have been. 


\section{Key Question: Frustrated?}

Family physicians report frustration about the barriers to improving cardiovascular risk factors among their patients, which they consider to be primarily outside of their control. ${ }^{14}$ Cost of care, lack of sufficient support from other health professionals, socioeconomic issues, and patients' lack of motivation are major barriers reported by family physicians. Crosson et $\mathrm{al}^{14}$ hypothesize that new motivational approaches that assist physicians in helping patients overcome these barriers could be the next steps to improving outcomes. Thankfully, many physicians already do help patients with these barriers.

\section{Key Question: Do Patients Have Adequate Health Literacy?}

Better health literacy is associated with better health. Health literacy is higher among those with more education, but unfortunately we cannot presume that even college graduates have good health literacy. Shah et $\mathrm{al}^{5}$ report on the use of a health literacy assessment instrument that takes $<3$ minutes, although that still seems like a lot of time in a busy office. The good news is that previously taking a health education class was associated with better health literacy, and this offers a potential solution. We should encourage health classes at multiple levels of education programs.

\section{Key Question for Pectus Excavatum: Short of breath? Low endurance?}

Pectus excavatum is frequently seen and varies from mild to severe. It is more common in men and in those with scoliosis. Psychosocial impairments are caused by this congenital anatomic phenomenon, as well as heart and lung function issues, which worsen with age. Surgery can provide significant relief and is best done during adolescence, but can be performed at any age. Jaroszewski et $\mathrm{al}^{12}$ provide us with pictures and more details.

\section{Key Technology: 3-Dimensional Ultrasound}

Forest et $\mathrm{al}^{11}$ present the first reported meningomyelocele detected during the first trimester of pregnancy. This report also may be unusual because it is from a family medicine office that has a 3-dimensional ultrasound machine, which is key to the detection of this entity.
Once again, we present a number of articles that advance the evidence-based practice of primary care. The "key question" strategy is a useful heuristic device to remind us that we are looking for patterns that can quickly lead us to potential answers; but these answers still must be evaluated for relevance with our individual patients.

Marjorie A. Bowman, MD, MPA

Anne Victoria Neale, $\mathrm{PhD}, \mathrm{MPH}$

\section{References}

1. Heisel MJ, Duberstein PR, Lyness JM, Feldman MD. Screening for suicide ideation among older primary care patients. J Am Board Fam Med 2010; 23:260-9.

2. Maghout-Juratli S, Janisse J, Schwartz K, Arnetz BB. The causal role of fatigue in the stress-perceived health relationship: a MetroNet study. J Am Board Fam Med 2010;23:212-9.

3. Kerr PL, Muehlenkamp JJ, Turner JM. Nonsuicidal self-injury: a review of current research for family medicine and primary care physicians. J Am Board Fam Med 2010;23:240-59.

4. Bluestein D, Rutledge CM, Healey AC. Psychosocial correlates of insomnia severity in primary care. J Am Board Fam Med 2010;23:204-11.

5. Ciccarelli Shah L, West P, Bremmeyr K, SavoyMoore RT. Health literacy instrument in family medicine: the "Newest Vital Sign" ease of use and correlates. J Am Board Fam Med 2010;23:195-203.

6. Shepherd AJ, Cass AR, Ray L. Determining risk of vertebral osteoporosis in men: validation of the Male Osteoporosis Risk Estimation Score. J Am Board Fam Med 2010;23:186-94.

7. Ferris D, Horn L, Waller JL. Parental acceptance of a mandatory human papillomavirus (HPV) vaccination program. J Am Board Fam Med 2010;23: 220-9.

8. Bender BG, Apter A, Bogen DK, et al. Test of an interactive voice response intervention to improve adherence to controller medications in adults with asthma. J Am Board Fam Med 2010;23:159-65.

9. McCoy EK, Thomas JL, Sowell RS, et al. An evaluation of peak expiratory flow monitoring: a comparison of sitting versus standing measurements. J Am Board Fam Med 2010;23:166-70.

10. Mulhem E, Kennedy EL, Lick D. Treatment of cervical dysplasia with the Fischer cone biopsy excisor in a family medicine office: a case series. J Am Board Fam Med 2010;23:154-8.

11. Forest CP, Goodman D, Hahn RG. Meningomyelocele: early detection using 3-dimensional ultrasound imaging in the family medicine center. J Am Board Fam Med 2010;23:270-2.

12. Jaroszewski D, Notrica D, McMahon L, Steidley DE, Deschamps C. Current management of pectus 
excavatum: a review and update of therapy and treatment recommendations. J Am Board Fam Med 2010; 23:230-9.

13. Winters P, Tancredi D, Fiscella K. The role of usual source of care in cholesterol treatment. J Am Board Fam Med 2010;23:179-85.
14. Crosson JC, Heisler M, Subramanian U, et al. Physicians' perceptions of barriers to cardiovascular disease risk factor control among patients with diabetes: results from the Translating Research into Action for Diabetes (TRIAD) study. J Am Board Fam Med 2010;23:171-8. 